\title{
Functional abdominal pain disorders in children: Bridging the gap
}

\section{Galle Medical Association Oration 2018}

\author{
Karunanayake A \\ Department of Physiology, Faculty of Medicine, University of Ruhuna, Galle, Sri Lanka.
}

Correspondence: Dr. A. Karunanayake

e-mail: a2222nath@gmail.com

https://orcid.org/0000-0001-5836-6329

In early twentieth century, Sir George Frederic Still, known as the father of British Paediatrics, wrote; "I know of no symptom which can be more obscure in its causation than colicky abdominal pain in childhood". Today, a century later, colicky abdominal pain in children still remains an symptom which is often difficult to understand and management is a challenge. Half a century later, Dr. J Apley, another British Paediatrician, studied abdominal pain among children extensively named this symptom complex as "recurrent abdominal pain syndrome of childhood"

He defined it as "at least three episodes of abdominal pain, severe enough to affect their activities over a period longer than three months" His findings formed the main guidelines for the paediatricians and researchers dealing with this problem for the next half of the century.

Dr. John Appley found that $10.5 \%$ of British schoolchildren were suffering from RAP in 1958. Since then it has been studied all over the world, including Asian countries, and has been reported to occur in $8-12 \%$ of school-aged children. According to previous epidemiological studies RAP is the second commonest painful health problem in schoolaged children, only second to a headache.

Several studies have shown $75 \%$ to $90 \%$ of the RAP patients are suffering from none- organic type of RAP. When a child presents with organic pain, the clinician may be able to objectively test for, and diagnose, a physical cause for that pain. This is not the case with non-organic pain, as there is no definitive test. The investigation, at best, will only exclude organic disease it will not prove a functional origin. Therefore, the diagnosis of non-organic pain abdominal pain is both intellectually and medically challenging.

The vast majority of children and adolescents with recurrent abdominal pain have functional gastro -intestinal disorders. Functional gastrointestinal disorders are defined as "chronic or recurrent gastrointestinal symptoms, which are not explained by structural or biochemical abnormalities". It is a spectrum of clinical presentation diagnosed by symptoms based on Rome criteria.

The road to the development of the Rome criteria for FGIDs began from a landmark meeting in Rome. In 1988, During the $12^{\text {th }}$ International Congress of Gastroenterology in Rome a working team was set up to create guidelines for the management for IBS. This decision has revolutionized the approach to FGIDs by deviating the previous "diagnoses of exclusion" approach and introducing the "diagnoses of inclusion" approach. The first edition of RomeRome I was published in 1994 which outline the symptom-based diagnostic criteria for 21 FGIDs. Rome I diagnostic criteria was revised and in 1999 Rome II was published. Rome II included the diagnostic criteria for paediatric FGIDs for the first time. Rome III was published in September 2006 which is a nearly thousand-page document written by a collaborative effort of 82 international experts. The Rome III classification includes 28 adult and 17 paediatric diagnostic entities. The new Rome IV which was released on May 2016, is evidence-based, multicultural oriented and with clinical applications.

There are four functional gastrointestinal disorders, which are presented as the abdominal pain as the predominant symptom. Therefore Irritable bowel syndrome, abdominal migraine, Functional 
dyspepsia, Functional abdominal pain are known as functional abdominal pain disorders. In Rome IV, the label of abdominal pain-related functional gastrointestinal disorders (AP-FGIDs) was replaced by the term functional abdominal pain disorders (FAPDs).

Despite the high prevalence, underlying pathophysiology of this condition is poorly understood and effective treatment options are lacking. During this presentation, I would like to discuss Epidemiology, risk factors pathophysiology, the impact of the disease. Finally the management of the functional abdominal pain in children.

\section{Epidemiology of FAPDs}

The first Part my presentation is dedicated to the epidemiology of functional abdominal pain disorders. A recent meta-analysis of 58 epidemiologic studies on abdominal pain conducted from 1957 to 2014, including nearly two hundred thousand children, reported a global pooled prevalence of $13.5 \%$. According to different continents, the pooled prevalence was more stable, though was slightly lower in European studies and generally higher in studies from South-America and Asia. Most studies conducted in Europe, Asia and the USA did not show significant association between the socioeconomic status and the disease. However very few studies have reported the prevalence of FAPDs in 5 to 12 age group. Therefore an epidemiological study was conducted to determined the Sri Lankan prevalence in 5 to 12 age group. The findings of the epidemiological study were published as abstracts in $5^{\text {th }}$ Asian neurogastroenterology and motility meeting in Osaka, Japan and The $14^{\text {th }}$ Asian Pan-Pacific Society for Pediatric Gastroenterology, Hepatology and Nutrition meeting in Bangkok, Thailand.

The study was carried out using a self-administered parental questionnaire in western province of Sri Lanka. We have used standard and internationally accepted tools in data collection. All questionnaires were translated, validated and pretested in the native language (Sinhala).

Main parts of the research questionnaire included,

Rome III questionnaire for paediatric functional gastrointestinal disorders
Family impact questionnaire [Preds QL Family Impact Module - Version 2]

$>$ Health-related quality of life inventory [Preds QL Paediatric Quality of life Inventory-version 4 for child report]

Child abuse questionnaire (self-report form for children older than 13 years)

Healthcare consultation details report

The questionnaires were filled with the help of the research assistants, which increased the accuracy and validity of the research. A total of 1000 children recruited from four randomly selected schools. $653(65.3 \%)$ correctly filled questionnaires were included in the analysis. The prevalence FAPDs was $12.6 \%$ in $5-12$ age group. There were 82 children who fulfilled criteria for the FAPDs.

FAPDs was significantly prevalent in females. The commonest FAPDs subtype was Functional abdominal pain followed by IBS. The Prevalence of an Abdominal migraine was less than $1 \%$ in our cohort.

The pain profile showed that majority of the children had

$>$ Moderate to severe pain, lasting less than one hour for less than 3 months duration.

$>$ Duration of abdominal pain was more than one year in $25 \%$ of children.

Duration of abdominal pain was significantly higher in females children

However, the severity of pain, duration of one pain episode and frequency of abdominal pain were not significantly different between males and females. Pain in another site, abdominal fullness and headache were the most common associated symptoms. However, there was no statistically significant difference between males and females with related to associated symptoms. Epigastric and periumbilical were the commonest sites for abdominal pain. However, most of the previous studies have reported the periumbilical area as the commonest site of abdominal. Higher prevalence of functional dyspepsia in this cohort who have epigastric pain may have contributed to the observed deviation in our study. 


\section{Risk factors and pathophysiological mechanisms in functional abdominal pain disorders}

Second part is dedicated on risk factors and pathophysiological mechanisms in functional abdominal pain disorders. Rome IV has several recognized groups of risk factors that are associated with FAPDs. It is suggested that most of them complexly blend with other identified pathophysiological mechanisms to potentiate their effects at both central to generate symptoms.

I have assessed gender, exposure to the child abuse, early life events, and genetic factors as risk factors. At $5^{\text {th }}$ Asian neurogastroenterology and motility meeting, Osaka, Japan abstract was presented with related to the risk factor I have studied. Previous, school-based epidemiological study have shown that except from five to six-year age group, females had a higher prevalence of FAPDs in our study. This dominance in females was reported in all different continents across the world. In addition to that, the predominance of females has been also described in other functional complaints, like functional constipation and headache. Effect of sex hormones and higher visceral sensitivity have been proposed as contributing factor for female predominance in
Functional pain. However, our sample includes children of a very young age who do not have a full female hormone profile. Therefore, the exact cause for the observed female predominance is not apparent in our study.

The findings related to the exposure to abuse as a risk factor were published in the Journal of Tropical Pediatrics which is indexed in science citation index .

This publication has received the national research council merit award for scientific publication.

School-based study was conducted in 13 - 18 aged school children by using self-administered child questionnaire. The written consent was obtained from parents. Assent was also obtained from children in addition to parental consent

A total of 1855 questionnaires were distributed and $1850(99.7 \%)$ properly filled questionnaires were included in the study.

The prevalence of FAPDs was significantly higher in children exposed to all three main types of abuse physical, emotional and sexual abuse (Table 1). Observation was noted across the all age groups we have assessed (Figure 1).

Table 1: Prevalence of FAPDs according to the type of abuse

\begin{tabular}{|c|c|c|c|c|c|c|c|c|}
\hline \multirow[b]{2}{*}{$\begin{array}{l}\text { Type of } \\
\text { AP-FGID }\end{array}$} & \multicolumn{2}{|c|}{ Physical abuse } & \multicolumn{2}{|c|}{ Emotional abuse } & \multicolumn{2}{|c|}{ Sexual abuse } & \multicolumn{2}{|c|}{ Any type of abuse } \\
\hline & $\begin{array}{c}\text { Yes } \\
n(\%)\end{array}$ & $\begin{array}{c}\text { No } \\
n(\%)\end{array}$ & $\begin{array}{c}\text { Yes } \\
n(\%)\end{array}$ & $\begin{array}{c}\text { No } \\
n(\%)\end{array}$ & $\begin{array}{c}\text { Yes } \\
n(\%)\end{array}$ & $\begin{array}{c}\text { No } \\
n(\%)\end{array}$ & $\begin{array}{c}\text { Yes } \\
n(\%)\end{array}$ & $\begin{array}{c}\text { No } \\
n(\%)\end{array}$ \\
\hline IBS & $36(7.7 \%)^{*}$ & $55(4.0 \%)$ & $37(8.5 \%)^{*}$ & $54 \quad(3.8 \%)$ & $10(18.9 \%)^{*}$ & $81(4.6 \%)$ & $59(8.5 \%)^{*}$ & $32(2.8 \%)$ \\
\hline FD & $5(1.1 \%)$ & $6(0.4 \%)$ & $3(0.7 \%)$ & $8 \quad(0.6 \%)$ & 0 & $11(0.6 \%)$ & $6 \quad(0.9 \%)$ & $5 \quad(0.4 \%)$ \\
\hline $\mathbf{A M}$ & $10 \quad(2.1 \%)$ & $27(1.9 \%)$ & $16(3.7 \%)^{*}$ & $21 \quad(1.5 \%)$ & $2 \quad(3.8 \%)$ & $35(2.0 \%)$ & $18(2.6 \%)^{*}$ & $19(1.6 \%)$ \\
\hline FAP & $49(10.4 \%)$ & $131(9.5 \%)$ & $61(14.0 \%)^{*}$ & $119(8.4 \%)$ & $8(15.1 \%)$ & $172(9.7 \%)$ & $82(11.8 \%)^{*}$ & $98 \quad(8.5 \%)$ \\
\hline AP-F & $95(20.2 \%)^{*}$ & $210(15.1 \%)$ & $109(25.0 \%)^{*}$ & $196(13.8 \%)$ & $18(34.0 \%)^{*}$ & $287(16.2 \%)$ & $155(22.4 \%)^{*}$ & $150(13.0 \%)$ \\
\hline
\end{tabular}
total

${ }^{*} p<0.05$ compared to not abuses, unpaired t-test

IBS = irritable bowel syndrome, FD = functional dyspepsia, $\mathrm{AM}=$ Abdominal migraine, $\mathrm{FAP}=$ functional abdominal pain, $\mathrm{AP}-\mathrm{FGD}=$ abdominal pain predominant functional gastrointestinal diseases

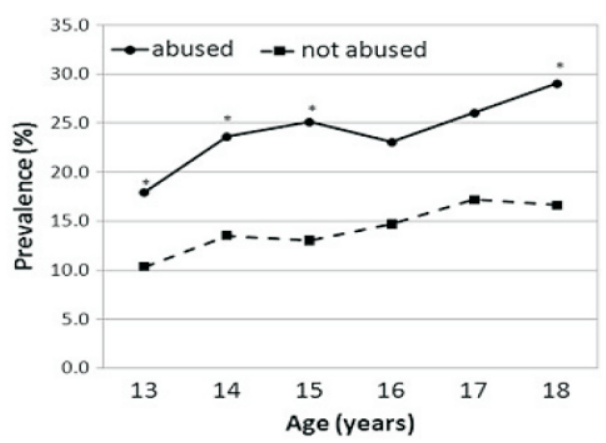

Figure 1: Prevalence of FAPDs according to the exposure of abuse 
In addition to that those with functional abdominal pain, exposed to abuse had a significantly higher severity score for bowel symptoms.

Very few researchers have studied the relationship between child abuse and functional pain disorders during the childhood, especially among teenagers. None of those previous studies has assessed the impact of abuse on symptom profile. This novel finding indicates the importance of child abuse in developing FAPDs. The Rome committee have used the result of this study and included child maltreatment as a risk factor in Rome IV guidelines.

Second risk factor I have assessed was early life events. Using a parental questionnaire, details of ELE were obtained from 182 children with FAPDs in the $5-12$ age group. 571 children were included in the control group. Prenatal complications were significantly higher in the FAPD group. Gestational diabetes and pregnancy-induced hypertension were the most common antenatal complications. Post-natal complications were also significantly higher in the FAPDs group. In addition to that receiving $\mathrm{PBU}$ care were significantly higher in the FAPDs group. The current study has added pre-natal and post-natal complications to the list of early life events associated with FAPDs. These findings signify the fact that adverse life events occurring during the fetal period and the neonatal period which is a vulnerable period for developing neurons may be an important contributory factor for the development of FAPDs. However, the duration of the gestational period was not different between FAPDs and control group. Another ELE significantly associated with FAPDs was low in the birth order. That means elder children in the family are more prone to develop the disease. Having a family member with a history of chronic abdominal or any other chronic pain was also significantly higher in FAPDs group. Adult studies have revealed a large number of genes associated with FAPDs especially with IBS and IBS patients are more likely to have a family history of similar illness. Our study has strengthen the idea of familial predisposition in FAPDs.

\section{Pathophysiology of FAPDs}

In this $3^{\text {rd }}$ part, I want to highlight the pathophysiological mechanism behind the symptom generation. Pathophysiology of FGIDs is a grey area in gastroenterology. Even with the highly advanced modern technologies, pathophysiological mechanisms of FGIDs are not yet clearly understood. The recognized pathophysiological mechanisms include

visceral hypersensitivity

$>$ dysmotility

$>$ immunological dysfunction

$>$ altered gastrointestinal microbiota

$>$ altered intestinal permeability

genetic factors

psychosocial disturbance

Rome IV has described FAPDs as a disorder of gutbrain interaction. However most of the proposed mechanisms do not correlate with the clinical symptoms.

I have studied the Gastric motility and autonomic functions as the pathophysiological mechanisms.

Motility studies have repeatedly shown Abnormalities in the gastrointestinal motor function as a potential pathophysiological mechanism in FAPDs. Dilated gastric antrum at fasting period, delayed gastric emptying, impaired initial distribution of a meal, impaired gastric accommodation to a meal and antral hypomotility have been reported as the abnormal motility patterns.

Autonomic nervous system is an integral part of the brain-gut axis that is involved in regulation of gastrointestinal motility. It is the one of the first mechanism investigated as a pathophysiology mechanism in functional pain. The Role of autonomic system in symptom generation is a controversial point. Available literature has shown that autonomic activity may present as normal, hypofunction or hyperactive status in functional abdominal pain in children. However, the relationship between autonomic function and gastric motility has not been studied in children. This phase of the study has been presented as four abstracts in $1^{\text {st }}$ federation of neurogastromotility meeting at Guangzhou, China, $5^{\text {th }}$ Asian neurogastroenterology and motility meeting, Osaka, Japan. A journal article is under review process in the World Journal of Gastroenterology as an invited article. 
We have recruited 100 children with FAPDs for the laboratory study. Diagnosis were confirmed with thorough clinical and investigations procedures. Their motility and autonomic parameters were compared with 50 age, sex-matched healthy children. We were adhered to strict laboratory protocols to minimize investigators bias, environmental and diurnal variational impact on those physiological parameters. After a test meal, same time of the day, gastric motility parameters were measured by u previously validate real-time USS method in using a high-resolution real-time scanner. All gastric motility parameters were assessed by the same investigator who was blind to the diagnosis and results of the autonomic function tests results. Seven motility parameters were measured including gastric emptying rate and motility index as main motility parameters.

Four bedside, non-invasive, autonomic test were conducted to assess autonomic functions which were described by Ewings. Those test were previously used and validated for children. All the tests were conducted in thermo-neutral conditions (26C) and at the same time of day (9.30 a.m. - 10.30 a.m.) in all the children. All the readings were recorded by a single observer to eliminate interpersonal bias.

Gastric motility results have shown that Gastric emptying rate, frequency of antral contractions, amplitude of antral contractions and motility index were significantly impaired in affected children (table 2)

In all four types of FAPDs main motility parameters gastric emptying rate and motility index were lower than the controls. There was a correlation between some motility parameters with pain parametres. In contrast to the motility, autonomic parameter were not significantly different between two groups (Table 3).

We have found that in the control group, several autonomic parameters were correlated with the motility.

This has indicated the intact physiological relationship between gut and brain in healthy children. In contrast to that, any of the autonomic parameters did not correlate with any of the motility parameters in FAPDs indicating affected children gut has failed to respond to the autonomic commands from the brain.
We named this phenomenon as functional extrinsic denervation in FAPDs

During the laboratory study I have investigated relationship between BMI and motility. Childhood obesity is a global pandemic. Several studies have shown the association between obesity and functional gastrointestinal disorders in children.

However, none of the studies have given a clear explanation for the development of functional gastrointestinal disorders in obese children. Therefore, we have assessed the Body mass index as a risk factor for FGIDs. Abstracts was presented at $7^{\text {th }}$ European paediatric gastrointestinal motility meeting at Sorrento Italy and annual academic session of PSSL. It has won the best paper award in annual academic session of PSSL.

It has found that Children with more than 15 BMI had significantly larger Fasting antral area, antral area in I min after a test meal and antral area in 15 min after a test meal. That indicates the presence of large stomach in both fasting and fed state in children with high BMI. Furthermore, positive correlation was observed in BMI and Antral area after 1 minute and Antral area after 15 minutes after the test meal. The Postprandial antral dilatation is indicated by antral area in I min after a test meal and antral area 15 minute after a test meal. Riezzo et al., has also found marked postprandial antral dilatation in dyspeptic children. Our finding of abnormalities in antral motility parameters propose a possible pathophysiological mechanism for the development ofFAPDs in obese children.

Based on laboratory observations, we have proposed a disease model named as automatic stomach in FAPDs to explain the symptom generation in FAPDs.

This model was presented in $5^{\text {th }}$ Asian neurogastroenterology and motility meeting, Osaka, Japan. Development of theoretical, etiopathogenic mechanisms involved automatic stomach and its consequences are shown in the figure 2 . 
Table 2: Comparison of gastric motility parameters between FAPDs and control groups

\begin{tabular}{|c|c|c|c|}
\hline Gastric motility parameter & $\begin{array}{c}\text { AP-FGIDs } \\
(n=100) \\
\text { Mean (SD) }\end{array}$ & $\begin{array}{c}\text { Controls } \\
(n=50) \\
\text { Mean (SD) }\end{array}$ & $p$ value* \\
\hline Fasting antral area $\left(\mathrm{cm}^{2}\right)$ & $\begin{array}{ll}1.7 \quad(0.9) \\
\end{array}$ & $1.6(0.9)$ & 0.34 \\
\hline Antral area in $1 \min \left(\mathrm{cm}^{2}\right)$ & $9.4 \quad(2.5)$ & $10.1(3.4)$ & 0.14 \\
\hline Antral area in $15 \mathrm{~min}\left(\mathrm{~cm}^{2}\right)$ & $5.1 \quad(1.9)$ & $5.4(10.5)$ & 0.80 \\
\hline Gastric emptying rate (\%) & $45.7(15.0)$ & $59.6(15.2)$ & $<0.0001$ \\
\hline Frequency of antral contraction (/3min) & $8.3 \quad(1.0)$ & $9.4 \quad(0.7)$ & $<0.0001$ \\
\hline Amplitude of antral contraction (\%) & $48.7(12.2)$ & $58.2(14.9)$ & $<0.0001$ \\
\hline Antral motility index & $4.1 \quad(1.2)$ & $6.4(6.6)$ & $<0.0001$ \\
\hline
\end{tabular}

Table 3: Comparison of autonomic parameters between FAPDs and control group

\begin{tabular}{|c|c|c|c|c|}
\hline Autonomic test & Measurement & $\begin{array}{l}\text { AP-FGIDs } \\
(\mathrm{n}=100) \\
\text { Mean (SD) }\end{array}$ & $\begin{array}{c}\text { Controls } \\
(n=50) \\
\text { Mean (SD) }\end{array}$ & $p$ value* \\
\hline Resting heart rate (beats/min) & $\begin{array}{l}\text { Resting heart rate } \\
\text { (beats/min) }\end{array}$ & $89.8(12.3)$ & $87.9(15.1)$ & 0.43 \\
\hline $\begin{array}{l}\text { Heart rate response to deep } \\
\text { breathing }\end{array}$ & $\begin{array}{l}\text { Maximum-minimum heart } \\
\text { rate (beats/min) }\end{array}$ & $31.3(10.9)$ & $31.8(11.5)$ & 0.79 \\
\hline $\begin{array}{l}\text { Lying to standing heart rate } \\
\text { response }\end{array}$ & $30: 15$ ratio & $1.22(0.2)$ & $1.26(0.2)$ & 0.51 \\
\hline Valsalva manoeuvre & Valsalva ratio & $1.5(0.3)$ & $1.6 \quad(0.3)$ & 0.17 \\
\hline Postural blood pressure change & Fall in SBP $(\mathrm{mmHg})$ & $-6.3(7.0)$ & $-2.6(8.6)$ & 0.21 \\
\hline
\end{tabular}

* Mann-Whitney $U$ test

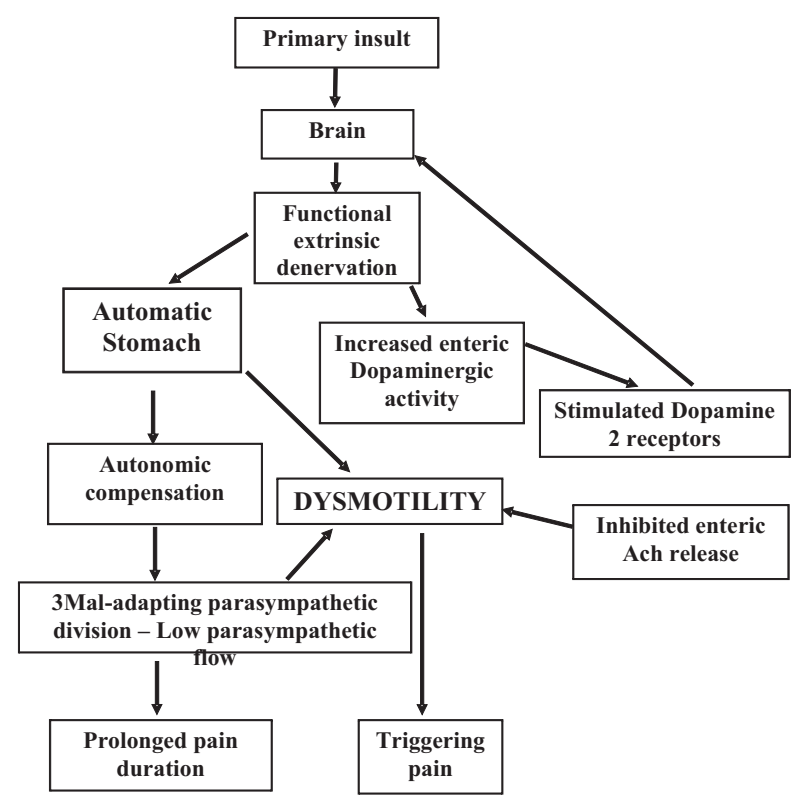

Figure 2: Development of theoretical, etiopathogenic mechanisms involved automatic stomach in FAPDs (Karunanayake, Rajindrajith, de Silva, Gunawardena Devanarayana, 2019) 
Primary insults diminishing the extrinsic (autonomic) control over the stomach lead to automatic stomach in susceptible children who may having altered physiological mechanism as a result of early life events. Stress, psychosocial factors or infection may be acting as a primary insult. The behaviour of the uncontrolled automatic stomach and inhibited Ach release via dopamine 2 receptors may lead to development dysmotility. Abdominal pain is triggered by the Motility abnormalities. In the process, compensatory mechanism may try to gain control. We have no evidence on this statement in the current study.

However, the high parasympathetic flow may be the autonomic compensation demonstrated in previous studies BY Jorgensen et al., 1993 . Parasympathetic system failing and maladapting as indicated by low parasympathetic flow. Parasympathetic maladaptation prolongs the duration of pain episode and may worsen the motility abnormalities. In a nutshell, Automatic stomach model would describe FAPDs are an "emerging rebel group when the local government (Gut) trying to get freedom from the empire (Brain)".

\section{Impact of FAPDs}

I will focus on the impact of FAPDs during the $3^{\text {rd }}$ segment of the presentation.

Chronic abdominal pain affects child life in many ways. Epidemiological studies have shown that 10\% to $15 \%$ of affected children have reported the recurrent and chronic nature affects their wellbeing. Several studies have clearly illustrated that FAPDs reduces quality of life of affected children. In addition to that, Sagawa and co-workers point out that some FAPDs affect quality of school work as well . Furthermore, one-third of the affected children continue to experience pain at least for five years. Finally, poorly managed children with FGIDs continue to have symptoms during adulthood and that is clearly linked with IBS in adults.

There is a very significant impact of FGIDs on health care systems.US Studies has shown that more than half of new pediatric gastrointestinal clinic patients met the Rome 3 criteria for more than 1 FGIDs and noted low yield from basic investigations. Functional dyspepsia had always been the most expensive FAPD to treat. Those data clearly illustrate the healthcare burden and rising costs of FAPDs on the health budgets of countries. During the previously described epidemiological study, impact of the disease on 5 to 12 age group was assessed by using the parental questionnaires. This phase of the study has been presented as four abstracts in $1^{\text {st }}$ federation of neurogastromotility meeting at Guangzhou, China, $5^{\text {th }}$ Asian neurogastroenterology and motility meeting, Osaka, Japan.

Some degree of disturbances to child activities due to abdominal pain was reported in nearly $80 \%$ of children with FAPDs. The majority of them reported to have disturbances in schooling. Sleep disturbances were recorded in $75 \%$ of the affected children. Anorexia was reported in $75 \%$ of children. Engaging in hobbies were the least affected activity which was $68.3 \%$. Both males and female children were equally affected in their daily activities. Health relate quality of life was assessed as the second indicator of the disease impact. Healthrelated quality of life (HRQOL) is a subjective, multidimensional concept to assess impact on disease. Child HRQOL is the final outcome of interactions with various social contexts surrounding him or her. The quality of life assessment scale we used (PedsQL) was composed of 23 items comprising four dimensions:

$$
\begin{aligned}
& \text { physical functioning } \\
& \text { emotional functioning } \\
& \text { social functioning } \\
& \text { school functioning }
\end{aligned}
$$

Total score is computed out of 100 . Higher scores indicate better functioning.

Our study has shown except social functioning domain all other domains and total HRQOL was significantly impaired in affected children. Although social functioning domain was low in FAPDs, it was not statistically significant. Social functioning is a measure to assess the individual's ability to maintain social relations. However, there is evidence to suggest that chronically ill children do not differ from their healthy peers in social adjustment as suggested by Spieth and Harris .

Family play a major role in the development in FGIDs in biopsychosocial model of FGIDs. Surprisingly, we have found no epidemiological studies investigating the family impact I 5 to 12 age group. For the first time, we have reported the impact 
of FGIDs on the family. The tool we used, The parent report of the PedsQL 2.0 family impact module was composed of 36 items comprising eight dimensions: Total score is computed out of 100 . Higher scores indicate less family impact.

Although FGIDs are benign diseases, parents of affected children have shown significantly lower scores which indicating a high family impact. Apart from daily activity and social domains, all other domains have shown lower scores compared with healthy children.

Burden on the health care system is an important factor in health economy. We have evaluated the health care consultations in FAPDs which is an indirect measure of the burden on health economy. Healthcare consultations were significantly higher in FAPDs group. In 5- 12 age group, 40\% FAPDs children had nearly 3 healthcare consultations during the past one year for abdominal pain. Multiple logistic regression analysis showed that nausea and presence of a family member with chronic pain was significantly associated with healthcare consultations. A negative correlation was observed between the total HRQOL score and healthcare consultations for abdominal pain. However, the majority of the children did not seek consultations for abdominal pain. It may an indicator of the prevalence of "silent suffers" in the community.

\section{Management of FAPDs}

In this Final part of my presentation I will focus on the management of FAPDs. As the first step for diagnostic workup, it is essential to devote adequate time for the history and physical examination as pathophysiological mechanisms underlying FAPDs remain unclear and currently no diagnostic biomarkers exist. History taking should include details of abdominal pain, infectious episodes or stressful events associated with the onset of symptoms, psychosocial history, dietary triggers, history of previous treatments, and family incidence of gastrointestinal diseases. Physical examination should include thorough general examination to identify evidence of an organic disease. Careful attention needs to be paid to growth parameters as well. Detailed abdominal examination, perianal, and rectal examination are also crucial in confirming the diagnosis of FAPDs. Only when alarm symptoms or so-called red flags are present which may indicate an organic disease diagnostic testing are recommended.

It has been demonstrated that numerous laboratory investigations are performed during common diagnostic workup of children with FAPDs, without detecting clinically meaningful abnormalities, but with additional inconvenience and cost. There is the possibility of finding some false positive investigations, and frequently treated for a disease that actually does not exist. The failure in acknowledging this was already playfully called Ulisses' syndrome, in allusion to the Greek mythological hero Ulysses fought in the Trojan war but afterwards took 10 years, with many dangerous and pointless adventures, before he got back to where he had started. Similarly, the unnecessary and uncritical use of laboratory examinations, leading to long investigation journeys, and making the child and his/her family go through an unnecessary, expensive, and sometimes dangerous expedition, whose end is the starting point. However When clinicians or families require further reassurance, few judicial investigations can be performed. When no atypical clinical features are present, abdominal ultrasonography does not have significant diagnostic value.

The goal of management of functional abdominal pain disorders in children and adolescents is return to normal function rather than complete elimination of pain. When symptoms persist and disrupt a child's wellbeing, pharmacological or nonpharmacological treatment should be considered.

Education, demystification, and reassurance play key roles in the management of FAPDs, starting with explanation of the diagnosis to the child and caregivers followed by Prevalence of FGDs, Benign clinical course, intermittent nature of symptoms. It is important to stress to the change parents response to child's pain

Most children outgrow symptoms. it is important hat physicians develop a positive therapeutic alliance with the patient and the family during the initial visit. This endeavour should be used to educate patients and parents on possible underlying pathophysiological mechanisms and reasonable expectations on treatment outcome.

Both pharmacological and non-pharmacological methods have been experimented on FGIDs. 
However Therapeutic options for FAPDs are often limited. It is apparent that Lack of understanding in the pathophysiology, heterogeneity of the diseases and multifactorial pathological mechanisms have contributed to non-availabilty of effective treatment modalities. Interventions such as amitriptyline has shown no benefits over placebo. Mebeverine, Famotidine, cyproheptadine, and rifaximin had only shown a modest effects and long term follow up data on treated children were not available. There is no convincing evidence that dietary interventions such as increasing the fibre content in the diet or a low FODMAP diet help in the management of childhood FAPDs. Other interventions such as guided imagery and hypnotherapy therapy are time-consuming and need specially trained professionals and therefore, difficult to implement in busy clinical settings. In such a context, finding a potentially effective, widely available, low-cost therapeutic agent has farreaching benefits to children.

We have conducted a randomized controlled clinical trial on value of domperidone in functional abdominal pain in children. The trial was published in Journal of Pediatric Gastroenterology and Nutrition which is indexed in science citation index .

Several factors favours our selection of domperidone as a therapeutic agent.

Repeated finding of gastrointestinal motor abnormalities among children with FAPDs. Favourable results were reported in adult studies. According to the proposed "Automatic stomach model, functional extrinsic denervation is affecting gastric motility by increasing the dopaminergic inhibition on the gastrointestinal motility via DAR2 receptors. Therefore Domperidone - dopamine 2 receptor blocker can block the one pathway leading to the dysmotility. Several previous studies have supported our idea to attack enteric dopaminergic system.

Consecutive patients belonging to 5 - 12 year age group who were eligible were recruited from paediatric outpatient clinics at teaching hospital, Ragama. All recruited patients were assessed by a Consultant Paediatrician. All patients were screened for organic diseases using history, examination (including growth parameters), stool microscopy, urine microscopy and culture, full blood count, Creactive protein, liver and renal function tests.
Special investigations performed based on findings of the initial evaluation and investigation in some patients included upper and lower endoscopy. A baseline electrocardiogram was also performed to rule out cardiac conduction abnormalities. Patients were not screened for coeliac disease and lactose intolerance since they are extremely rare in Sri Lanka. Children and parents were instructed not to change the diet or lifestyle of the child once the subjects were included into the trial.

All 100 children with AP- FGIDs recruited were randomized, using computer-generated random numbers, into two groups (50 in a group) irrespective of the symptom severity and gastric motility status. The intervention group received domperidone $10 \mathrm{mg}$ (brand name Motilium ${ }^{\circledR}$ ) 3 times per day, 30 minutes before meals for 8 weeks. The control group received a placebo in same dosing regimen for the same duration. The placebo was identical to domperidone tablet in physical appearance and taste. A diary was provided to document adherence to treatment, severity, frequency and duration of symptoms and interruption of activities. Primary outcomes were measured at the beginning, completion of treatment course ( 8 weeks) and at 6 months. Secondary outcomes were measured before treatment and at the end of 8 weeks. Both patients, parents and investigators were blind for the treatments and investigation results. Ethical approval for this study was obtained from the Ethical Review Committee of the Faculty of Medicine, University of Kelaniya. The trial was registered in the Sri Lanka Clinical Trial Registry. Two primary and two secondary outcomes were assessed.

Primary outcomes measured was cure and patient reported general improvement .

Cure was defined as,

Abdominal pain episodes less than 4 per month,

Severity of abdominal pain is less than $25 \mathrm{~mm}$ in the visual analogue scale,

No interruption of activities due to abdominal pain

Improvement was defined as overall satisfaction and satisfactory relief.

Decrease in pain severity and increase in gastric motility were measured secondary outcomes. 
We have assessed 162 patients. Based on inclusion and exclusion criteria we have recruited 100 children for the study. 50 patients were allocated for each arm of the study. At the end of 8 weeks of treatment 89 patients completed the trial. We followed them up to 6 months and 79 patients responded for $6^{\text {th }}$ month follow up. This response rate at the end of $8^{\text {th }}$ week and $6^{\text {th }}$ months have given adequate power for analysis. Data were analysed using intention to teat analysis. According to the analysis after 8 week of treatment children treated with domperidone have shown significant improvement. At the end of $6^{\text {th }}$ month, both cure and improvement rate were significantly higher in domperidone group. Significant improvement were seen in pain reduction and motility index as the secondary outcome in domperidone (table 4).

And we compared the primary and secondary outcomes in domperidone group according to the baseline motility parameters (table 5).
When primary outcomes were compared between children with normal motility and those with abnormal motility, percentage with cure and improvement of overall condition were not significantly different after administration of domperidone at 8 weeks and 6 months. When secondary outcomes were compared, reduction in pain severity was not different between the two groups. Domperidone resulted in significant improvement of gastric emptying rate and antral motility index in children with normal gastric motility. Therefore domperidone can be prescribed irrespective of the motility status. Safety and adverse effects during the trial also important.

One patient developed a skin rash during the trail. This presentation was not considered as an adverse effect of treatment. The child completed the trial without further problems. No treatment-associated adverse events were noted during the trial period.

Table 4: Primary and secondary outcomes after interventions in $8^{\text {th }}$ week and $6^{\text {th }}$ month

\begin{tabular}{|c|c|c|c|c|c|}
\hline \multicolumn{2}{|l|}{ Outcome } & \multicolumn{2}{|c|}{$\begin{array}{c}\text { Domperidone group } \\
(n=50)\end{array}$} & $\begin{array}{l}\text { Placebo group } \\
\qquad(n=50)\end{array}$ & \multirow[t]{2}{*}{$p$ value } \\
\hline Primary outcome & At 8 weeks & & & & \\
\hline & Cure & 22 & $(44.0)$ & $14 \quad(28.0)$ & $0.096^{*}$ \\
\hline & Improvement & 37 & $(74.0)$ & $25 \quad(50.0)$ & $0.013^{*}$ \\
\hline & At 6 months & & & & \\
\hline & Cure & 30 & $(60.0)$ & $19 \quad(38.0)$ & $0.028^{*}$ \\
\hline & Improvement & 44 & $(88.0)$ & $33 \quad(66.0)$ & $0.009^{*}$ \\
\hline \multirow[t]{4}{*}{ Secondary outcome } & At $8^{\text {th }}$ week & & & & \\
\hline & $\%$ reduction of pain severity & 54.1 & $(35.8)$ & $29.7(50.2)$ & $0.008 \dagger$ \\
\hline & $\%$ improvement in gastric emptying & 14.8 & (7.6) & $7.4(11.2)$ & $0.423 \dagger$ \\
\hline & $\%$ improvement in antral motility index & 27.5 & (5.3) & $7.2(4.4)$ & $0.029 \dagger$ \\
\hline
\end{tabular}

$\uparrow$ Independent- sample $t$ test

Table 5: Primary and secondary outcomes after 8 weeks of interventions according to the baseline motility parameters

\begin{tabular}{|c|c|c|c|c|c|c|}
\hline \multirow[t]{2}{*}{ Outcome } & \multicolumn{2}{|c|}{ Domperidone } & \multirow[t]{2}{*}{$p$ value } & \multicolumn{2}{|c|}{ Placebo } & \multirow[t]{2}{*}{$p$ value } \\
\hline & $\begin{array}{c}\text { Normal } \\
\text { motility }(n=26)\end{array}$ & $\begin{array}{c}\text { Low motility } \\
(n=24)\end{array}$ & & $\begin{array}{c}\text { Normal motility } \\
\quad(n=22)\end{array}$ & $\begin{array}{c}\text { Low motility } \\
\quad(n=28)\end{array}$ & \\
\hline \multicolumn{7}{|l|}{ Primary out come } \\
\hline Cure & $13(50.0)$ & $9(37.5)$ & $0.374 *$ & $5(22.7)$ & $9(32.1)$ & $0.462 *$ \\
\hline Improvement & $20(76.9)$ & $17(70.8)$ & $0.624 *$ & $10(45.4)$ & $11(39.2)$ & $0.369 *$ \\
\hline \multicolumn{7}{|l|}{ Secondary out come } \\
\hline$\%$ reduction of pain severity & 75.8 & 65.2 & 0.402 & -56.6 & -41.4 & $0.412 \dagger$ \\
\hline$\%$ improvement in gastric emptying & 55.6 & 39.4 & 0.001 & -10.8 & 34.9 & $0.410 \dagger$ \\
\hline$\%$ improvement in antral motility index & 5.1 & 4.3 & 0.037 & 4.06 & 4.38 & $0.627 \dagger$ \\
\hline
\end{tabular}




\section{Conclusions}

In conclusion,

The FAPDs is a common disorder with female predisposition in 5-12 age group.

Impact of AP-FGIDs are severe enough ,

$>$ to reduce health-related quality of life in affected children.

$>$ to increase health care seeking behaviour and cause a significant impact on the families of affected children.

Early life events are an important risk factor.

Main gastric motility parameters assessed were significantly lower in children with FAPDs. Assessment of autonomic functions in FAPDs show neither a significant difference compared to the control group nor a correlation with gastric motility abnormalities. Extensive investigations are of limited value in diagnosing. Because proposed pathophysiological mechanisms are usually not revealed by usual investigations. Domperidone, a prokinetic drug, has shown promising, long-lasting therapeutic value in management of functional abdominal pain in children. Therapeutic value of domperidone is not related to baseline gastric motility status. Therefore it can be prescribe without performing motility studies.

\section{Acknowledgements}

I wish to express my sincere gratitude to all parents and children participated in the study. Their feedback was constructive and contributed to the success of the study.

I am bound to thank Prof. Niranga Devanarayana, Professor of Physiology and Prof. Shaman Rajindrajith, Professor of Paediatrics, University of Kelaniya, Prof. Sampath Gunawardena, Professor in Physiology, University of Ruhuna and Prof. Asita de Silva, Senior Professor of Pharmacology, University of Kelaniya for all the support extended to me.

I would also like to thank Mrs. Janeshwari Liyanage and Mrs. Janaki Ariyawansa, Technical officers of the Gastroenterology Research Laboratory, Faculty of Medicine, University of Kelaniya, for their immense support for motility studies.
My wholehearted gratitude goes to my wife and the two children for their cooperation and patience during the study.

\section{References}

1. Devanarayana NM, Rajindrajith S, Perera MS, Nishanthanie SW, Karunanayake A, Benninga MA. Association between functional gastrointestinal diseases and exposure to abuse in teenagers. J Trop Pediatr, 2014, 60(5): 386-392. doi: 10.1093/tropej/fmu035.

2. Jorgensen LS, Christiansen P, Raundahl U, Ostgaard S, Christensen NJ, Fenger M, Flachs H. Autonomic nervous system function in patients with functional abdominal pain. An experimental study. Scand J Gastroenterol, 1993; 28(1): 63-65.

3. Karunanayake A, Devanarayana NM, de Silva A, Gunawardena S, Rajindrajith S. Randomized Controlled Clinical Trial on Value of Domperidone in Functional Abdominal Pain in Children. $J$ Pediatr Gastroenterol Nutr, 2018; 66(5), 725-731. doi: 10.1097/mpg. 0000000000001819.

4. Karunanayake A, Rajindrajith S, de Silva HA, Gunawardena S, Devanarayana NM. Autonomic functions and gastric motility in children with functional abdominal pain disorders. World J Gastroenterol, 2019; 25(1): 95-106. doi: 10.3748/wjg.v25.i1.95.

5. Riezzo G, Chiloiro M, Guerra V, Borrelli O, Salvia G, Cucchiara S. Comparison of gastric electrical activity and gastric emptying in healthy and dyspeptic children. Dig Dis Sci, 2000; 45(3): 517-524.

6. Sagawa T, Okamura S, Kakizaki S, Zhang Y, Morita K, Mori M. Functional gastrointestinal disorders in adolescents and quality of school life. J Gastroenterol Hepatol, 2013; 28(2): 285-290. doi: 10.1111/j.1440-1746.2012.07257.

7. Spieth LE, Harris CV. Assessment of Health-Related Quality of Life in Children and Adolescents: An Integrative Review. J Pediatr Psychol, 1996; 21(2): 175-193. doi: 10.1093/jpepsy/21.2.175. 